\title{
Choosing Products in Social Networks
}

\author{
Sunil Simon * Krzysztof R. Apt ${ }^{\dagger}$
}

\begin{abstract}
We study the consequences of adopting products by agents who form a social network. To this end we use the threshold model introduced in [2], in which the nodes influenced by their neighbours can adopt one out of several alternatives, and associate with each such social network a strategic game between the agents. The possibility of not choosing any product results in two special types of (pure) Nash equilibria.

We show that such games may have no Nash equilibrium and that determining the existence of a Nash equilibrium, also of a special type, is NP-complete. The situation changes when the underlying graph of the social network is a DAG, a simple cycle, or has no source nodes. For these three classes we determine the complexity of establishing whether a (special type of) Nash equilibrium exists.

We also clarify for these categories of games the status and the complexity of the finite improvement property (FIP). Further, we introduce a new property of the uniform FIP which is satisfied when the underlying graph is a simple cycle, but determining it is co-NP-hard in the general case and also when the underlying graph has no source nodes. The latter complexity results also hold for verifying the property of being a weakly acyclic game.
\end{abstract}

\section{Introduction}

\subsection{Background}

Social networks are a thriving interdisciplinary research area with links to sociology, economics, epidemiology, computer science, and mathematics. A flurry of numerous articles and recent books, see, e.g., [7], testifies to the relevance of this field. It deals with such diverse topics as epidemics, analysis of the connectivity, spread of certain patterns of social behaviour, effects of advertising, and emergence of 'bubbles' in financial markets.

One of the prevalent types of models of social networks are the threshold models introduced in [8. In such a setup each node $i$ has a threshold $\theta(i) \in(0,1]$ and adopts an 'item' given in advance (which can be a disease, trend, or a specific

\footnotetext{
${ }^{*}$ Centre for Mathematics and Computer Science (CWI)

${ }^{\dagger}$ Centre for Mathematics and Computer Science (CWI) and ILLC, University of Amsterdam, The Netherlands
} 
product) when the total weight of incoming edges from the nodes that have already adopted this item exceeds the threshold. One of the most important issues studied in the threshold models has been that of the spread of an item, see, e.g., [14, 11, 6]. From now on we shall refer to an 'item' that is spread by a more specific name of a 'product'.

In this context very few papers dealt with more than one product. One of them is [9] with its focus on the notions of compatibility and bilinguality that result when one adopts both available products at an extra cost. Another one is [4], where the authors investigate whether the algorithmic approach of [1] can be extended to the case of two products.

In 2 the authors introduced a new threshold model of a social network in which nodes (agents) influenced by their neighbours can adopt one out of several products. This model allowed us to study various aspects of the spread of a given product through a social network, in the presence of other products. We analysed from the complexity point of view the problems of determining whether adoption of a given product by the whole network is possible (respectively, necessary), and when a unique outcome of the adoption process is guaranteed. We also clarified for social networks without unique outcomes the complexity of determining whether a given node has to adopt some (respectively, a given) product in some (respectively, all) final network(s), and the complexity of computing the minimum and the maximum possible spread of a given product.

\subsection{Motivation}

Our interest here is in understanding and predicting the behaviour of the consumers (agents) who form a social network and are confronted with several alternatives (products). To carry out such an analysis we use the above model of [2] and associate with each such social network a natural strategic game. In this game the strategies of an agent are products he can choose. Additionally a 'null' strategy is available that models the decision of not choosing any product. The idea is that after each agent chose a product, or decided not to choose any, the agents assess the optimality of their choices comparing them to the choices made by their neighbours. This leads to a natural study of (pure) Nash equilibria, in particular of those in which some, respectively all, constituent strategies are non-null.

Social network games are related to graphical games of [10, in which the payoff function of each player depends only on a (usually small) number of other players. In this work the focus was mainly on finding mixed (approximate) Nash equilibria. However, in graphical games the underlying structures are undirected graphs. Also, social network games exhibit the following join the crowd property: the payoff of each player depends only on his strategy and on the set of players who chose his strategy and weakly increases when more players choose his strategy.

Since these games are related to social networks, some natural special cases are of interest: when the underlying graph is a DAG, has no source nodes or a simple cycle which is a special case of a graph without source nodes. Such social 
networks correspond respectively to a hierarchical organization or to a 'circle of friends', in which everybody has a friend (a neighbour). Studying Nash equilibria of these games and various properties defined in terms of improvement paths allows us to gain better insights into the consequences of adopting products.

\subsection{Related work}

There are a number of papers that focus on games associated with various forms of networks, see, e.g., [15] for an overview. A more recent example is 1] that analyses a strategic game between players being firms who select nodes in an undirected graph in order to advertise competing products via 'viral marketing'. However, in spite of the focus on similar questions concerning the existence and structure of Nash equilibria and on their reachability, from a technical point of view, the games studied here seem to be unrelated to the games studied elsewhere.

Still, it is useful to mention the following phenomenon. When the underlying graph of a social network has no source nodes, the game always has a trivial Nash equilibrium in which no agent chooses a product. A similar phenomenon has been recently observed in [5] in the case of their network formation games, where such equilibria are called degenerate. Further, note that the 'join the crowd' property is exactly the opposite of the defining property of the congestion games with player-specific payoff functions introduced in [12. In these game the payoff of each player weakly decreases when more players choose his strategy. Because in our case (in contrast to [12]) the players can have different strategy sets, the resulting games are not coordination games.

\section{Preliminaries}

\subsection{Strategic games}

Assume a set $\{1, \ldots, n\}$ of players, where $n>1$. A strategic game for $n$ players, written as $\left(S_{1}, \ldots, S_{n}, p_{1}, \ldots, p_{n}\right)$, consists of a non-empty set $S_{i}$ of strategies and a payoff function $p_{i}: S_{1} \times \ldots \times S_{n} \rightarrow \mathbb{R}$, for each player $i$.

Fix a strategic game $G:=\left(S_{1}, \ldots, S_{n}, p_{1}, \ldots, p_{n}\right)$. We denote $S_{1} \times \cdots \times S_{n}$ by $S$, call each element $s \in S$ a joint strategy, denote the $i$ th element of $s$ by $s_{i}$, and abbreviate the sequence $\left(s_{j}\right)_{j \neq i}$ to $s_{-i}$. We also write $\left(s_{i}, s_{-i}\right)$ instead of $s$. We call a strategy $s_{i}$ of player $i$ a best response to a joint strategy $s_{-i}$ of his opponents if $\forall s_{i}^{\prime} \in S_{i} p_{i}\left(s_{i}, s_{-i}\right) \geq p_{i}\left(s_{i}^{\prime}, s_{-i}\right)$. Next, we call a joint strategy $s$ a Nash equilibrium if each $s_{i}$ is a best response to $s_{-i}$, that is, if

$$
\forall i \in\{1, \ldots, n\} \forall s_{i}^{\prime} \in S_{i} p_{i}\left(s_{i}, s_{-i}\right) \geq p_{i}\left(s_{i}^{\prime}, s_{-i}\right) .
$$

Given a joint strategy $s$ we call the sum $S W(s)=\sum_{j=1}^{n} p_{j}(s)$ the social welfare of $s$. When the social welfare of $s$ is maximal we call $s$ a social optimum. Recall that, given a finite game that has a Nash equilibrium, its price of anarchy (respectively, price of stability) is the ratio $\frac{S W(s)}{S W\left(s^{\prime}\right)}$ where 
$s$ is a social optimum and $s^{\prime}$ is a Nash equilibrium with the lowest (respectively, highest) social welfare. For division by zero, we interpret the outcome as $\infty$.

Next, we call a strategy $s_{i}$ of player $i$ a better response given a joint strategy $s$ if $p_{i}\left(s_{i}^{\prime}, s_{-i}\right)>p_{i}\left(s_{i}, s_{-i}\right)$. Following the terminology of [13], a path in $S$ is a sequence $\left(s^{1}, s^{2}, \ldots\right)$ of joint strategies such that for every $k>1$ there is a player $i$ such that $s^{k}=\left(s_{i}^{\prime}, s_{-i}^{k-1}\right)$ for some $s_{i}^{\prime} \neq s_{i}^{k-1}$. A path $\xi$ is called an improvement path if it is maximal and for all $k$ smaller than the length of $\xi, p_{i}\left(s^{k}\right)>p_{i}\left(s^{k-1}\right)$, where $i$ is the player who deviated from $s^{k-1}$. The last condition simply means that each deviating player selects a better response. A game has the finite improvement property (in short, FIP) if every improvement path is finite. Obviously, if a game has the FIP, then it has a Nash equilibrium-the last element of each path.

Finally, recall that a game is called weakly acyclic (see [12]) if for every joint strategy there exists a finite improvement path that starts at it.

\subsection{Social networks}

We are interested in specific strategic games defined over social networks. In what follows we focus on a model of social networks recently introduced in [2].

Let $V=\{1, \ldots, n\}$ be a finite set of agents and $G=(V, E, w)$ a weighted directed graph with $w_{i j} \in[0,1]$ being the weight of the edge $(i, j)$. We often use the notation $i \rightarrow j$ to denote $(i, j) \in E$ and write $i \rightarrow^{*} j$ if there is a path from $i$ to $j$ in the graph $G$. Given a node $i$ of $G$ we denote by $N(i)$ the set of nodes from which there is an incoming edge to $i$. We call each $j \in N(i)$ a neighbour of $i$ in $G$. We assume that for each node $i$ such that $N(i) \neq \emptyset, \sum_{j \in N(i)} w_{j i} \leq 1$. An agent $i \in V$ is said to be a source node in $G$ if $N(i)=\emptyset$.

Let $\mathcal{P}$ be a finite set of alternatives or products. By a social network (from now on, just network) we mean a tuple $\mathcal{S}=(G, \mathcal{P}, P, \theta)$, where $P$ assigns to each agent $i$ a non-empty set of products $P(i)$ from which it can make a choice. $\theta$ is a threshold function that for each $i \in V$ and $t \in P(i)$ yields a value $\theta(i, t) \in(0,1]$.

Given a network $\mathcal{S}$ we denote by source $(\mathcal{S})$ the set of source nodes in the underlying graph $G$. One of the classes of the networks we shall study are the ones with $\operatorname{source}(\mathcal{S})=\emptyset$.

\subsection{Social network games}

Fix a network $\mathcal{S}=(G, \mathcal{P}, P, \theta)$. Each agent can adopt a product from his product set or choose not to adopt any product. We denote the latter choice by $t_{0}$.

With each network $\mathcal{S}$ we associate a strategic game $\mathcal{G}(\mathcal{S})$. The idea is that the nodes simultaneously choose a product or abstain from choosing any. Subsequently each node assesses his choice by comparing it with the choices made by his neighbours. Formally, we define the game as follows: the players are the agents, the set of strategies for player $i$ is $S_{i}:=P(i) \cup\left\{t_{0}\right\}$, for $i \in V, t \in P(i)$

and a joint strategy $s$, let $\mathcal{N}_{i}^{t}(s):=\left\{j \in N(i) \mid s_{j}=t\right\}$, i.e., $\mathcal{N}_{i}^{t}(s)$ is the set of 
neighbours of $i$ who adopted in $s$ the product $t$. The payoff function is defined as follows, where $c_{0}$ is some positive constant given in advance:

- for $i \in \operatorname{source}(\mathcal{S}), p_{i}(s):=\left\{\begin{array}{lll}0 & \text { if } & s_{i}=t_{0} \\ c_{0} & \text { if } & s_{i} \in P(i)\end{array}\right.$

- for $i \notin \operatorname{source}(\mathcal{S}), p_{i}(s):= \begin{cases}0 & \text { if } s_{i}=t_{0} \\ \sum_{j \in \mathcal{N}_{i}^{t}(s)} w_{j i}-\theta(i, t) & \text { if } s_{i}=t, \text { for some } t \in P(i)\end{cases}$

Let us explain the underlying motivations behind the above definition. In the first entry we assume that the payoff function for the source nodes is constant only for simplicity. In the last section of the paper we explain that the obtained results hold equally well in the case when the source nodes have arbitrary positive utility for each product.

The second entry in the payoff definition is motivated by the following considerations. When agent $i$ is not a source node, his 'satisfaction' from a joint strategy depends positively from the accumulated weight (read: 'influence') of his neighbours who made the same choice as him, and negatively from his threshold level (read: 'resistance') to adopt this product. The assumption that $\theta(i, t)>0$ reflects the view that there is always some resistance to adopt a product. So when this resistance is high, it can happen that the payoff is negative. Of course, in such a situation not adopting any product, represented by the strategy $t_{0}$, is a better alternative.

The presence of this possibility allows each agent to refrain from choosing a product. This refers to natural situations, such as deciding not to purchase a smartphone or not going on vacation. In the last section we refer to an initiated research on social network games in which the strategy $t_{0}$ is not present. Such games capture situations in which the agents have to take some decision, for instance selecting a secondary school for their children.

By definition the payoff of each player depends only on the strategies chosen by his neighbours, so the social network games are related to graphical games of 10 . However, the underlying dependence structure of a social network game is a directed graph and the presence of the special strategy $t_{0}$ available to each player makes these games more specific.

In what follows for $t \in \mathcal{P} \cup\left\{t_{0}\right\}$ we use the notation $\bar{t}$ to denote the joint strategy $s$ where $s_{j}=t$ for all $j \in V$. This notation is legal only if for all agents $i$ it holds that $t \in P(i)$. The presence of the strategy $t_{0}$ motivates the introduction and study of special types of Nash equilibria. A Nash equilibrium $s$ is

- determined if for all $i, s_{i} \neq t_{0}$,

- non-trivial if for some $i, s_{i} \neq t_{0}$,

- trivial if for all $i, s_{i}=t_{0}$, i.e., $s=\overline{t_{0}}$. 


\section{Nash equilibria: general case}

The first natural question that we address is that of the existence of Nash equilibria in the social network games. We establish the following result.

Theorem 1. Deciding whether for a network $\mathcal{S}$ the game $\mathcal{G}(\mathcal{S})$ has a (respectively, non-trivial) Nash equilibrium is NP-complete.

To prove it we first construct an example of a social network game with no Nash equilibrium and then use it to determine the complexity of the existence of Nash equilibria.

Example 2. Consider the network given in Figure 1, where the product set of each agent is marked next to the node denoting it and the weights are labels on the edges. The source nodes are represented by the unique product in the product set.

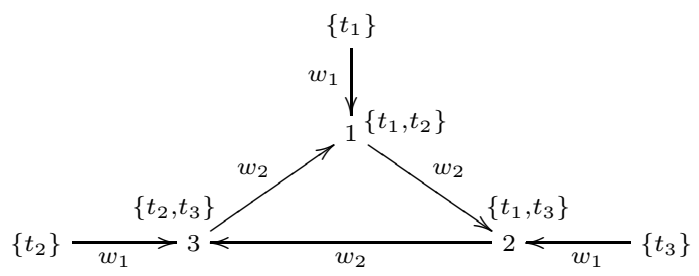

Figure 1: A network with no Nash equilibrium

So the weights on the edges from the nodes $\left\{t_{1}\right\},\left\{t_{2}\right\},\left\{t_{3}\right\}$ are marked by $w_{1}$ and the weights on the edges forming the triangle are marked by $w_{2}$. We assume that each threshold is a constant $\theta$, where $\theta<w_{1}<w_{2}$. So it is more profitable to a player residing on a triangle to adopt the product adopted by his neighbour residing on a triangle than by the other neighbour who is a source node. For convenience we represent each joint strategy as a triple of strategies of players 1,2 and 3 .

It is easy to check that in the game associated with this network no joint strategy is a Nash equilibrium. Indeed, each agent residing on the triangle can secure a payoff of at least $w_{1}-\theta>0$, so it suffices to analyze the joint strategies in which $t_{0}$ is not used. There are in total eight such joint strategies. Here is their listing, where in each joint strategy we underline the strategy that is not a best response to the choice of other players: $\left(t_{1}, t_{1}, t_{2}\right),\left(t_{1}, t_{1}, \underline{t_{3}}\right),\left(t_{1}, t_{3}, \underline{t_{2}}\right)$, $\left(t_{1}, \underline{t_{3}}, t_{3}\right),\left(t_{2}, \underline{t_{1}}, t_{2}\right),\left(t_{2}, \underline{t_{1}}, t_{3}\right),\left(t_{2}, t_{3}, \underline{t_{2}}\right),\left(\underline{t_{2}}, t_{3}, t_{3}\right)$.

Proof of Theorem [1. As in [2, to show NP-hardness, we use a reduction from the NP-complete PARTITION problem, which is: given $n$ positive rational numbers $\left(a_{1}, \ldots, a_{n}\right)$, is there a set $S$ such that $\sum_{i \in S} a_{i}=\sum_{i \notin S} a_{i}$ ? Consider an instance $I$ of PARTITION. Without loss of generality, suppose we have normalised the numbers so that $\sum_{i=1}^{n} a_{i}=1$. Then the problem instance sounds: does there exist a set $S$ such that $\sum_{i \in S} a_{i}=\sum_{i \notin S} a_{i}=\frac{1}{2}$ ? 
To construct the appropriate network we employ the networks given in Figure 1 and in Figure 2, where for each node $i \in\{1, \ldots, n\}$ we set $w_{i a}=w_{i b}=a_{i}$, and assume that the threshold of the nodes $a$ and $b$ is constant and equal $\frac{1}{2}$.

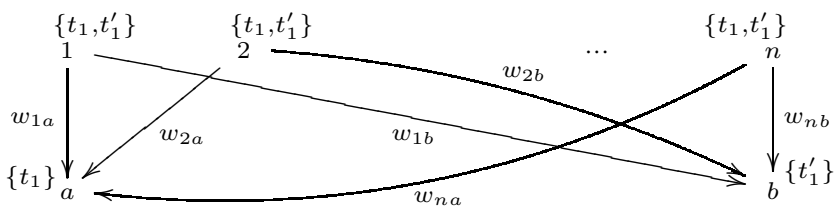

Figure 2: A network related to the PARTITION problem

We use two copies of the network given in Figure 11, one unchanged and the other in which the product $t_{1}$ is replaced by $t_{1}^{\prime}$, and construct the desired network $\mathcal{S}$ by identifying the node $a$ of the network from Figure 2 with the node marked by $\left\{t_{1}\right\}$ in the network from Figure 1 , and the node $b$ with the node marked by $\left\{t_{1}^{\prime}\right\}$ in the modified version of the network from Figure 1 .

Suppose now that a solution to the considered instance of the PARTITION problem exists, i.e., for some set $S \subseteq\{1, \ldots, n\}$ we have $\sum_{i \in S} a_{i}=\sum_{i \notin S} a_{i}=$ $\frac{1}{2}$. Consider the game $\mathcal{G}(\mathcal{S})$ and the joint strategy formed by the following strategies:

- $t_{1}$ assigned to each node $i \in S$ in the network from Figure 2,

- $t_{1}^{\prime}$ assigned to each node $i \in\{1, \ldots, n\} \backslash S$ in the network from Figure 2,

- $t_{0}$ assigned to the nodes $a, b$ and the nodes 1 in both versions of the network from Figure 1 .

- $t_{3}$ assigned to the nodes 2, 3 in both versions of the networks from Figure1 and the two nodes marked by $\left\{t_{3}\right\}$,

- $t_{2}$ assigned to the nodes marked by $\left\{t_{2}\right\}$.

We claim that this joint strategy is a non-trivial Nash equilibrium. Consider first the player (i.e, node) $a$. The accumulated weight of its neighbours who chose strategy $t_{1}$ is $\frac{1}{2}$, so its payoff after switching to the strategy $t_{1}$ is 0 . Therefore $t_{0}$ is indeed a best response for player $a$. For the same reason, $t_{0}$ is also a best response for player $b$. The analysis for the other nodes is straightforward.

Conversely, suppose that a joint strategy $s$ is a Nash equilibrium in the game $\mathcal{G}(\mathcal{S})$. Then it is also a non-trivial Nash equilibrium. We claim that the strategy selected by the node $a$ in $s$ is $t_{0}$. Otherwise, this strategy equals $t_{1}$ and the strategies selected by the nodes of the network of Figure 1 form a Nash equilibrium in the game associated with this network. This yields a contradiction with our previous analysis of this network.

So $t_{0}$ is a best response of the node $a$ to the strategies of the other players chosen in $s$. This means that $\sum_{i \in\{1, \ldots, n\} \mid s_{i}=t_{1}} w_{i a} \leq \frac{1}{2}$. By the same reasoning 
$t_{0}$ is a best response of the node $b$ to the strategies of the other players chosen in $s$. This means that $\sum_{i \in\{1, \ldots, n\} \mid s_{i}=t_{1}^{\prime}} w_{i b} \leq \frac{1}{2}$.

But $\sum_{i=1}^{n} a_{i}=1$ and for $i \in\{1, \ldots, n\}, w_{i a}=w_{i b}=a_{i}$, and $s_{i} \in\left\{t_{1}, t_{1}^{\prime}\right\}$. So both above inequalities are in fact equalities. Consequently for $S:=\{i \in$ $\left.\{1, \ldots, n\} \mid s_{i}=t_{1}\right\}$ we have $\sum_{i \in S} a_{i}=\sum_{i \notin S} a_{i}$. In other words, there exists a solution to the considered instance of the PARTITION problem.

To prove that the problem lies in NP it suffices to notice that given a network $\mathcal{S}=(G, \mathcal{P}, P, \theta)$ with $n$ nodes checking if a joint strategy is a non-trivial Nash equilibrium can be done by means of $n \cdot|\mathcal{P}|$ checks, so in polynomial time.

\section{Nash equilibria: special cases}

In view of the fact that in general Nash equilibria may not exist we now consider networks with special properties of the underlying directed graph. We consider first networks whose underlying graph is a directed acyclic graph (DAG). Intuitively, such networks correspond to hierarchical organizations.

Theorem 3. Consider a network $\mathcal{S}$ whose underlying graph is a DAG.

(i) $\mathcal{G}(\mathcal{S})$ always has a non-trivial Nash equilibrium.

(ii) Deciding whether $\mathcal{G}(\mathcal{S})$ has a determined Nash equilibrium is NP-complete.

Theorem 4. Consider a network $\mathcal{S}=(G, \mathcal{P}, P, \theta)$ whose underlying graph is a simple cycle. There is a procedure that runs in time $\mathcal{O}(|\mathcal{P}| \cdot n)$, where $n$ is the number of nodes in $G$, that decides whether $\mathcal{G}(\mathcal{S})$ has a non-trivial (respectively, determined) Nash equilibrium.

Theorem 5. The price of anarchy and the price of stability for the games associated with the networks whose underlying graph is a DAG or a simple cycle is unbounded.

Finally, we consider the case when the underlying graph $G=(V, E)$ of a network $\mathcal{S}$ has no source nodes, i.e., for all $i \in V, N(i) \neq \emptyset$. Intuitively, such a network corresponds to a 'circle of friends': everybody has a friend (a neighbour). For such networks we prove the following result.

Theorem 6. Consider a network $\mathcal{S}=(G, \mathcal{P}, P, \theta)$ whose underlying graph has no source nodes. There is a procedure that runs in time $\mathcal{O}\left(|\mathcal{P}| \cdot n^{3}\right)$, where $n$ is the number of nodes in $G$, that decides whether $\mathcal{G}(\mathcal{S})$ has a non-trivial Nash equilibrium.

The proof of Theorem [6 requires some characterization results that are of independent interest. The following concept plays a crucial role. Here and elsewhere we only consider subgraphs that are induced and identify each such subgraph with its set of nodes. (Recall that $\left(V^{\prime}, E^{\prime}\right)$ is an induced subgraph of $(V, E)$ if $V^{\prime} \subseteq V$ and $E^{\prime}=E \cap\left(V^{\prime} \times V^{\prime}\right)$.)

We say that a (non-empty) strongly connected subgraph (in short, SCS) $C_{t}$ of $G$ is self sustaining for a product $t$ if for all $i \in C_{t}$, 
- $t \in P(i)$,

- $\sum_{j \in N(i) \cap C_{t}} w_{j i} \geq \theta(i, t)$.

An easy observation is that if $\mathcal{S}$ is a network with no source nodes, then it always has a trivial Nash equilibrium, $\overline{t_{0}}$. The following lemma states that for such networks every non-trivial Nash equilibrium satisfies a structural property which relates it to the set of self sustaining SCSs in the underlying graph. We use the following notation: for a joint strategy $s$ and product $t, \mathcal{A}_{t}(s):=\{i \in$ $\left.V \mid s_{i}=t\right\}$ and $P(s):=\left\{t \mid \exists i \in V\right.$ with $\left.s_{i}=t\right\}$.

Lemma 7. Let $\mathcal{S}=(G, \mathcal{P}, P, \theta)$ be a network whose underlying graph has no source nodes. If $s \neq \overline{t_{0}}$ is a Nash equilibrium in $\mathcal{G}(\mathcal{S})$ then for all products $t \in P(s) \backslash\left\{t_{0}\right\}$ and $i \in \mathcal{A}_{t}(s)$ there exists a self sustaining $S C S C_{t} \subseteq \mathcal{A}_{t}(s)$ for $t$ and $j \in C_{t}$ such that $j \rightarrow^{*} i$.

Lemma 8. Let $\mathcal{S}=(G, \mathcal{P}, P, \theta)$ be a network whose underlying graph has no source nodes. The joint strategy $\overline{t_{0}}$ is a unique Nash equilibrium in $\mathcal{G}(\mathcal{S})$ iff there does not exist a product $t$ and a self sustaining $S C S C_{t}$ for $t$ in $G$.

Proof. $(\Leftarrow)$ By Lemma 7 .

$(\Rightarrow)$ Suppose there exists a self sustaining SCS $C_{t}$ for a product $t$. Let $R$ be the set of nodes reachable from $C_{t}$ which eventually can adopt product $t$. Formally, $R:=\bigcup_{m \in \mathbb{N}} R_{m}$ where

- $R_{0}:=C_{t}$,

- $R_{m+1}:=R_{m} \cup\left\{j \mid t \in P(j)\right.$ and $\left.\sum_{k \in N(j) \cap R_{m}} w_{k j} \geq \theta(j, t)\right\}$.

Let $s$ be the joint strategy such that for all $j \in R$, we have $s_{j}=t$ and for all $k \in V \backslash R$, we have $s_{k}=t_{0}$. It follows directly from the definition of $R$ that $s$ satisfies the following properties:

(P1) for all $i \in V, s_{i}=t_{0}$ or $s_{i}=t$,

(P2) for all $i \in V, s_{i} \neq t_{0}$ iff $i \in R$,

(P3) for all $i \in V$, if $i \in R$ then $p_{i}(s) \geq 0$.

We show that $s$ is a Nash equilibrium. Consider first any $j$ such that $s_{j}=t$ (so $s_{j} \neq t_{0}$ ). By $(\mathrm{P} 2) j \in R$ and by $(\mathrm{P} 3) p_{j}(s) \geq 0$. Since $p_{j}\left(s_{-j}, t_{0}\right)=$ $0 \leq p_{j}(s)$, player $j$ does not gain by deviating to $t_{0}$. Further, by $(\mathrm{P} 1)$, for all $k \in N(j), s_{k}=t$ or $s_{k}=t_{0}$ and therefore for all products $t^{\prime} \neq t$ we have $p_{j}\left(s_{-j}, t^{\prime}\right)<0 \leq p_{j}(s)$. Thus player $j$ does not gain by deviating to any product $t^{\prime} \neq t$ either.

Next, consider any $j$ such that $s_{j}=t_{0}$. We have $p_{j}(s)=0$ and from (P2) it follows that $j \notin R$. By the definition of $R$ we have $\sum_{k \in N(j) \cap R} w_{k j}<\theta(j, t)$. Thus $p_{j}\left(s_{-j}, t\right)<0$. Moreover, for all products $t^{\prime} \neq t$ we also have $p_{j}\left(s_{-j}, t^{\prime}\right)<0$ for the same reason as above. So player $j$ does not gain by a unilateral deviation. We conclude that $s$ is a Nash equilibrium. 
For a product $t \in \mathcal{P}$, we define the set $X_{t}:=\bigcap_{m \in \mathbb{N}} X_{t}^{m}$, where

- $X_{t}^{0}:=\{i \in V \mid t \in P(i)\}$,

- $X_{t}^{m+1}:=\left\{i \in V \mid \sum_{j \in N(i) \cap X_{t}^{m}} w_{j i} \geq \theta(i, t)\right\}$.

The following characterization leads to a direct proof of the claimed result.

Lemma 9. Let $\mathcal{S}$ be a network whose underlying graph has no source nodes. There exists a non-trivial Nash equilibrium in $\mathcal{G}(\mathcal{S})$ iff there exists a product $t$ such that $X_{t} \neq \emptyset$.

Proof. Suppose $\mathcal{S}=(G, \mathcal{P}, P, \theta)$.

$(\Rightarrow)$ It follows directly from the definitions that if there is a self sustaining SCS $C_{t}$ for product $t$ then $C_{t} \subseteq X_{t}$. Suppose now that for all $t, X_{t}=\emptyset$. Then for all $t$, there is no self sustaining SCS for $t$. So by Lemma $8, \overline{t_{0}}$ is a unique Nash equilibrium.

$(\Leftarrow)$ Suppose there exists $t$ such that $X_{t} \neq \emptyset$. Let $s$ be the joint strategy defined as follows:

$$
s_{i}:= \begin{cases}t & \text { if } i \in X_{t} \\ t_{0} & \text { if } i \notin X_{t}\end{cases}
$$

By the definition of $X_{t}$, for all $i \in X_{t}, p_{i}(s) \geq 0$. So no player $i \in X_{t}$ gains by deviating to $t_{0}$ (as then his payoff would become 0 ) or to a product $t^{\prime} \neq t$ (as then his payoff would become negative since no player adopted $t^{\prime}$ ). Also, by the definition of $X_{t}$ and of the joint strategy $s$, for all $i \notin X_{t}$ and for all $t^{\prime} \in P(i)$, $p_{i}\left(t^{\prime}, s_{-i}\right)<0$. Therefore, no player $i \notin X_{t}$ gains by deviating to a product $t^{\prime}$ either. It follows that $s$ is a Nash equilibrium.

Proof of Theorem 6. On the account of Lemma 9, the following procedure can be used to check for the existence of a non-trivial Nash equilibrium.

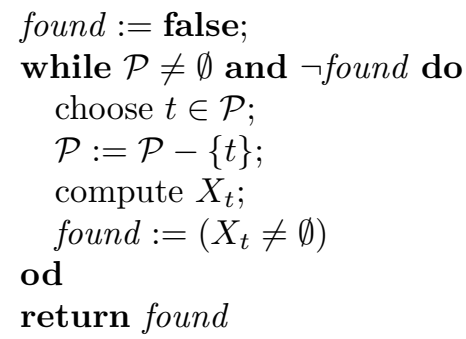

To assess its complexity, note that for a network $\mathcal{S}=(G, \mathcal{P}, P, \theta)$ and a fixed product $t$, the set $X_{t}$ can be constructed in time $\mathcal{O}\left(n^{3}\right)$, where $n$ is the number of nodes in $G$. Indeed, each iteration of $X_{t}^{m}$ requires at most $\mathcal{O}\left(n^{2}\right)$ comparisons and the fixed point is reached after at most $n$ steps. In the worst case, we need to compute $X_{t}$ for every $t \in \mathcal{P}$, so the procedure runs in time $\mathcal{O}\left(|\mathcal{P}| \cdot n^{3}\right)$. In

fact, the proof of Lemma 9 shows that if a non-trivial Nash equilibrium exists, then it can be constructed in polynomial time as well. 


\section{The FIP and the uniform FIP}

A natural question is whether the games for which we established the existence of a Nash equilibrium belong to some well-defined class of strategic games, for instance, games with the finite improvement property (FIP). When the underlying graph of the network is a DAG, the game does indeed have the FIP. The following theorem shows that the result can be improved in the case of two player social network games.

Theorem 10. Every two players social network game has the FIP.

Proof. By the above comment on DAGs, we can assume that the underlying graph is a cycle, say $1 \rightarrow 2 \rightarrow 1$. Consider an improvement path $\rho$. Without loss of generality we can assume that the players alternate their moves in $\rho$. In what follows given an element of $\rho$ (that is not the last one) we underline the strategy of the player who moves, i.e., selects a better response. We call each element of $\rho$ of the type $(\underline{t}, t)$ or $(t, \underline{t})$ a match. Further, we shorten the statement "each time player $i$ switches his strategy his payoff strictly increases and it never decreases when his opponent switches strategy" to "player $i$ 's payoff steadily goes up".

Consider two successive matches in $\rho$, based respectively on the strategies $t$ and $t_{1}$. The corresponding segment of $\rho$ is one of the following four types.

Type 1. $(\underline{t}, t) \Rightarrow^{*}\left(t_{1}, t_{1}\right)$. The fragment of $\rho$ that starts at $(\underline{t}, t)$ and finishes at $\left(\underline{t_{1}}, t_{1}\right)$ has the form: $(\underline{t}, t) \Rightarrow\left(t_{2}, \underline{t}\right) \Rightarrow^{*}\left(t_{1}, \underline{t_{3}}\right) \Rightarrow\left(\underline{t_{1}}, t_{1}\right)$. Then player 1's payoff steadily goes up. Additionally, in the step $\left(t_{1}, t_{3}\right) \rightrightarrows\left(t_{1}, t_{1}\right)$ his payoff increases by $w_{21}$. In turn, in the step $(\underline{t}, t) \Rightarrow\left(t_{2}, \underline{t}\right)$ player 2 's payoff decreases by $w_{12}$ and in the remaining steps his payoff steadily goes up. So $p_{1}(\bar{t})+w_{21}<p_{1}\left(\overline{t_{1}}\right)$ and $p_{2}(\bar{t})-w_{12}<p_{2}\left(\overline{t_{1}}\right)$.

Type 2. $(\underline{t}, t) \Rightarrow^{*}\left(t_{1}, \underline{t}_{1}\right)$. Then player 1 's payoff steadily goes up. In turn, in the first step of $(\underline{t}, t) \Rightarrow^{*}\left(t_{1}, t_{1}\right)$ the payoff of player 2 decreases by $w_{12}$, while in the last step (in which player 1 moves) his payoff increases by $w_{12}$. So these two payoff changes cancel against each other. Additionally, in the remaining steps player 2's payoff steadily goes up. So $p_{1}(\bar{t})<p_{1}\left(\overline{t_{1}}\right)$ and $p_{2}(\bar{t})<p_{2}\left(\overline{t_{1}}\right)$.

Type 3. $(t, \underline{t}) \Rightarrow^{*}\left(\underline{t_{1}}, t_{1}\right)$. This type is symmetric to Type 2 , so $p_{1}(\bar{t})<p_{1}\left(\overline{t_{1}}\right)$ and $p_{2}(\bar{t})<p_{2}\left(\overline{t_{1}}\right)$.

Type 4. $(t, \underline{t}) \Rightarrow^{*}\left(t_{1}, \underline{t_{1}}\right)$. This type is symmetric to Type 1 , so $p_{1}(\bar{t})-w_{21}<$ $p_{1}\left(\overline{t_{1}}\right)$ and $p_{2}(\bar{t})+w_{12}<p_{2}\left(\overline{t_{1}}\right)$.

Table 1 summarizes the changes in the payoffs between the two matches.

Consider now a match $(\underline{t}, t)$ in $\rho$ and a match $\left(\underline{t_{1}}, t_{1}\right)$ that appears later in $\rho$. Let $T_{i}$ denote the number of internal segments of type $i$ that occur in the fragment of $\rho$ that starts with $(\underline{t}, t)$ and ends with $\left(\underline{t_{1}}, t_{1}\right)$.

Case 1. $T_{1} \geq T_{4}$. Then Table 1 shows that the aggregate increase in $p_{1}$ in segments of type 1 exceeds the aggregate decrease in segments of type 4 . So $p_{1}(\bar{t})<p_{1}\left(\overline{t_{1}}\right)$.

Case 2. $T_{1}<T_{4}$. Then analogously Table 1 shows that $p_{2}(\bar{t})<p_{2}\left(\overline{t_{1}}\right)$.

We conclude that $t \neq t_{1}$. By symmetry the same conclusion holds if the considered matches are of the form $(t, \underline{t})$ and $\left(t_{1}, \underline{t_{1}}\right)$. This proves that each 


\begin{tabular}{|l|l|l|}
\hline Type & $p_{1}$ & $p_{2}$ \\
\hline 1 & $\begin{array}{l}\text { increases } \\
\text { by }>w_{21}\end{array}$ & $\begin{array}{l}\text { decreases } \\
\text { by }<w_{12}\end{array}$ \\
\hline 2,3 & increases & increases \\
\hline 4 & $\begin{array}{l}\text { decreases } \\
\text { by }<w_{21}\end{array}$ & $\begin{array}{l}\text { increases } \\
\text { by }>w_{12}\end{array}$ \\
\hline
\end{tabular}

Table 1: Changes in $p_{1}$ and $p_{2}$

match occurs in $\rho$ at most once. So in some suffix $\eta$ of $\rho$ no match occurs. But each step in $\eta$ increases the social welfare, so $\eta$ is finite, and so is $\rho$.

The FIP ceases to hold when the underlying graph has cycles. Figure 3(a) gives an example. Take any threshold and weight functions which satisfy the condition that an agent gets positive payoff when he chooses the product picked by his unique predecessor in the graph. Figure 3(b) then shows an infinite improvement path. In each joint strategy, we underline the strategy that is not a best response to the choice of other players. Note that at each step of this improvement path a best response is used. On the other hand, one can check that for any initial joint strategy there exists a finite improvement path. This is an instance of a more general result proved below.

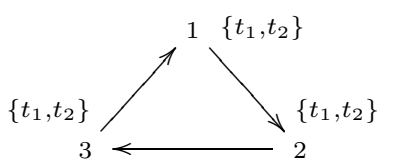

(a)

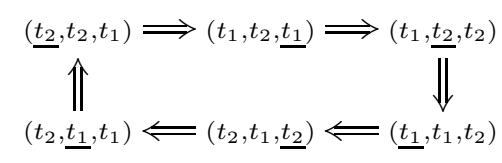

(b)

Figure 3: A social network with an infinite improvement path

By a scheduler we mean a function $f$ that given a joint strategy $s$ that is not a Nash equilibrium selects a player who did not select in $s$ a best response. An improvement path $\xi=s^{1}, s^{2}, \ldots$ conforms to a scheduler $f$ if for all $k$ smaller than the length of $\xi, s^{k+1}=\left(s_{i}^{\prime}, s_{-i}^{k}\right)$, where $f\left(s^{k}\right)=i$. We say that a strategic game has the uniform $\boldsymbol{F I P}$ if there exists a scheduler $f$ such that all improvement paths $\rho$ which conform to $f$ are finite. The property of having the uniform FIP is stronger than that of being weakly acyclic, see [3].

Theorem 11. Let $\mathcal{S}$ be a network such that the underlying graph is a simple cycle. Then the game $\mathcal{G}(\mathcal{S})$ has the uniform FIP.

Proof. We use the scheduler $f$ that given a joint strategy $s$ chooses the smallest index $i$ such that $s_{i}$ is not a best response to $s_{-i}$. So this scheduler selects a player again if he did not switch to a best response. Therefore we can assume that each selected player immediately selects a best response. 
Consider a joint strategy $s$ taken from a 'best response' improvement path. Observe that for all $k$ if $s_{k} \in P(k)$ and $p_{k}(s) \geq 0$ (so in particular if $s_{k}$ is a best response to $s_{-k}$ ), then $s_{k}=s_{k \ominus 1}$. So for all $i>1$, the following property holds:

$Z(i)$ : if $f(s)=i$ and $s_{i-1} \in P(i-1)$ then for all $j \in\{n, 1, \ldots, i-1\}, s_{j}=s_{i-1}$.

In words: if $i$ is the first player who did not choose a best response and player $i-1$ strategy is a product, then this product is a strategy of every earlier player and of player $n$. Along each 'best response' improvement path that conforms to $f$ the value of $f(s)$ strictly increases until the path terminates or at certain stage $f(s)=n$. In the latter case if $s_{n-1}=t_{0}$, then the unique best response for player $n$ is $t_{0}$. Otherwise $s_{n-1} \in P(n-1)$, so on the account of property $Z(n)$ all players' strategies equal the same product and the payoff of player $n$ is negative (since $f(s)=n$ ). So the unique best response for player $n$ is $t_{0}$, as well.

This switch begins a new round with player 1 as the next scheduled player. Player 1 also switches to $t_{0}$ and from now on every consecutive player switches to $t_{0}$, as well. The resulting path terminates once player $n-2$ switches to $t_{0}$.

\section{Concluding remarks}

In this paper we studied the consequences of adopting products by agents who form a social network. To this end we analysed a natural class of strategic games associated with the class of social networks introduced in [2]. The following table summarizes our complexity and existence results, where we refer to the underlying graph with $n$ nodes.

\begin{tabular}{|c|c|c|c|c|}
\hline property & arbitrary & DAG & simple cycle & $\begin{array}{c}\text { no source } \\
\text { nodes }\end{array}$ \\
\hline Arbitrary NE & NP-complete & always exists & always exists & always exists \\
Non-trivial NE & NP-complete & always exists & $\mathcal{O}(|\mathcal{P}| \cdot n)$ & $\mathcal{O}\left(|\mathcal{P}| \cdot n^{3}\right)$ \\
Determined NE & NP-complete & NP-complete & $\mathcal{O}(|\mathcal{P}| \cdot n)$ & $\mathrm{NP}$-complete \\
FIP & co-NP-hard & yes & - & co-NP-hard \\
Uniform FIP & co-NP-hard & yes & yes & co-NP-hard \\
Weakly acyclic & co-NP-hard & yes & yes & co-NP-hard \\
\hline
\end{tabular}

In the definition of the social network games we took a number of simplifying assumptions. In particular, we stipulated that the source nodes have a constant payoff $c_{0}>0$. One could allow the source nodes to have arbitrary positive utility for different products. This would not affect any proofs. Indeed, in the Nash equilibria the source nodes would select only the products with the highest payoff, so the other products in their product sets could be disregarded. Further, the FIP, the uniform FIP and weak acyclicity of a social network game is obviously not affected by such a modification.

The results of this paper can be slightly generalized by using a more general notion of a threshold that would also depend on the set of neighbours who 
adopted a given product. In this more general setup for $i \in V, t \in P(i)$ and $X \subseteq N(i)$, the threshold function $\theta$ yields a value $\theta(i, t, X) \in(0,1]$ and satisfies the following monotonicity condition: if $X_{1} \subseteq X_{2}$ then $\theta\left(i, t, X_{1}\right) \geq$ $\theta\left(i, t, X_{2}\right)$. Intuitively, agent $i$ 's resistance to adopt a product decreases when the set of its neighbours who adopted it increases. We decided not to use this definition for the sake of readability.

This work can be pursued in a couple of natural directions. One is the study of social networks with other classes of underlying graphs. Another is an investigation of the complexity results for other classes of social networks, in particular for the equitable ones, i.e., networks in which the weight functions

are defined as $w_{i j}=\frac{1}{|N(i)|}$ nodes $i$ and $j \in N(i)$. One could also consider other equilibrium concepts like the strict Nash equilibrium.

Finally, we also initiated a study of slightly different games, in which the players are obliged to choose a product, so the games in which the strategy $t_{0}$ is absent. Such games naturally correspond to situations in which the agents always choose a product, for instance a subscription for their mobile telephone. These games substantially differ from the ones considered here. For example, Nash equilibrium may not exist when the underlying graph is a simple cycle.

\section{References}

[1] N. Alon, M. Feldman, A. D. Procaccia, and M. Tennenholtz. A note on competitive diffusion through social networks. Inf. Process. Lett., 110(6):221$225,2010$.

[2] K. R. Apt and E. Markakis. Diffusion in social networks with competing products. In Proc. 4th International Symposium on Algorithmic Game Theory (SAGT11), volume 6982 of Lecture Notes in Computer Science, pages 212-223. Springer, 2011.

[3] K. R. Apt and S. Simon. A classification of weakly acyclic games. In Proc. 5th International Symposium on Algorithmic Game Theory (SAGT12), volume 7615 of Lecture Notes in Computer Science, pages 1-12. Springer, 2012.

[4] A. Borodin, Y. Filmus, and J. Oren. Threshold models for competitive influence in social networks. In Proc. 6th International Workshop on Internet and Network Economics (WINE 2010), pages 539-550, 2010.

[5] M. Brautbar and M. Kearns. A clustering coefficient network formation game. In Proc. 4th International Symposium on Algorithmic Game Theory (SAGT11), volume 6982 of Lecture Notes in Computer Science, pages 224235. Springer, 2011.

[6] N. Chen. On the approximability of influence in social networks. SIAM J. Discrete Math., 23(3):1400-1415, 2009.

[7] D. Easley and J. Kleinberg. Networks, Crowds, and Markets. Cambridge University Press, 2010. 
[8] M. Granovetter. Threshold models of collective behavior. American Journal of Sociology, 83(6):1420-1443, 1978.

[9] N. Immorlica, J. M. Kleinberg, M. Mahdian, and T. Wexler. The role of compatibility in the diffusion of technologies through social networks. In J. K. MacKie-Mason, D. C. Parkes, and P. Resnick, editors, ACM Conference on Electronic Commerce, pages 75-83. ACM, 2007.

[10] M. Kearns, M. Littman, and S. Singh. Graphical models for game theory. In Proceedings of the 17th Conference in Uncertainty in Artificial Intelligence (UAI '01), pages 253-260. Morgan Kaufmann, 2001.

[11] D. Kempe, J. M. Kleinberg, and É. Tardos. Maximizing the spread of influence through a social network. In L. Getoor, T. E. Senator, P. Domingos, and C. Faloutsos, editors, KDD, pages 137-146. ACM, 2003.

[12] I. Milchtaich. Congestion games with player-specific payoff functions. Games and Economic Behaviour, 13:111-124, 1996.

[13] D. Monderer and L. S. Shapley. Potential games. Games and Economic Behaviour, 14:124-143, 1996.

[14] S. Morris. Contagion. The Review of Economic Studies, 67(1):57-78, 2000.

[15] É. Tardos and T. Wexler. Network formation games and the potential function method. In N. Nisan, T. Roughgarden, É. Tardos, and V. J. Vazirani, editors, Algorithmic Game Theory, chapter 19, pages 487-516. Cambridge University Press, 2007. 